\title{
छs \\ New technique to measure emittance for beams with space charge
}

\author{
K. Poorrezaei, R. B. Fiorito, R. A. Kishek, and B. L. Beaudoin \\ Institute for Research in Electronics and Applied Physics, University of Maryland, College Park, Maryland 20742, USA
}

(Received 12 March 2013; published 16 August 2013)

\begin{abstract}
The characterization of the transverse phase space of beams is a fundamental requirement for particle accelerators. We present a novel approach for measurement of transverse emittance for beams with space charge, an important quality indicator of transverse phase space. The method utilizes a lens-drift-screen setup similar to that of a quadrupole scan emittance measurement. Measurements of radius and divergence that can be obtained from beam produced radiation, e.g. optical transition, are used to calculate the crosscorrelation term and therefore the rms emittance. A linear space-charge model is used in the envelope equations; hence, the errors in the measurement relate to the nonuniformity of the beam distribution. The emittance obtained with our method shows small deviation from those obtained by WARP simulations for beams with high space charge, in contrast to other techniques.
\end{abstract}

DOI: 10.1103/PhysRevSTAB.16.082801

\section{INTRODUCTION}

It is important to measure the transverse beam emittance in accelerators to quantify the beam quality and match the optics in an accelerator beam line. Most beams of interest are space-charge-dominated near the source and low energy transport section, where the beam dynamics are mostly determined by interparticle forces rather than the beam pressure represented by emittance. Space charge usually modifies and degrades the performance of emittance measurement methods such as quadrupole scan techniques [1-5]. Reference [6] presents a comprehensive analysis of space-charge force effects on such measurements.

The quadrupole scan (or "quad-scan") is one of the simplest and most common methods used to measure emittance. In a quad-scan, the emittance can be deduced from measurements of beam radius as a function of the strength of a quadrupole upstream. Since space-charge forces are a function of the beam radius, space charge confounds the analysis because an initial beam distribution has to be assumed a priori in order to calculate the space-charge term. In the case of extreme space charge, quad-scans become ineffective since the beam radius is determined largely by space charge, and wide variations in emittance lead to negligible change in beam radius. Several amendments have been proposed in literature to enhance the accuracy of the quadrupole scan method for high intensity beams $[7,8]$.

From the symmetry of the expression for the rms emittance [9],

Published by the American Physical Society under the terms of the Creative Commons Attribution 3.0 License. Further distribution of this work must maintain attribution to the author(s) and the published article's title, journal citation, and DOI.
PACS numbers: 29.27.Bd, 41.85.Ew, 41.85.Ja, 41.75.Ht

$$
\tilde{\boldsymbol{\epsilon}}^{2}=\left\langle x^{2}\right\rangle\left\langle x^{\prime 2}\right\rangle-\left\langle x \cdot x^{\prime}\right\rangle^{2}
$$

it is obviously possible to determine emittance with a quadrupole scan technique using measurements of either the beam radius or divergence. The usual implementation of the method is to perform a quadrupole scan of beam size only. However, by measurement of both the near field and the far field angular distribution of optical radiation produced by interaction of the beam with foils (transition radiation), magnetic fields (synchrotron or edge radiation), or apertures (diffraction radiation), it is possible to obtain simultaneous, high-quality measurements of beam size and divergence [10-13]. In this paper, we propose a method for measuring emittance, assuming a linear space-charge model, from a small number of measurements of divergence and radius.

In order to use these observables to determine the rms emittance at focus points other than a beam waist condition, which is not necessarily the same as a beam size minimum obtained by magnetic focusing, we need a methodology which relates the measured observables, i.e., divergence and beam size to the cross-correlation $\left(\left\langle x \cdot x^{\prime}\right\rangle\right)$ term. We will show how this can be done by taking the cross-correlation term as a control variable to match beam envelopes to their actual envelopes with the constraint that the beam radii and divergences at the screen are the same as measured values. First, we show that when space charge is negligible, emittance for either the horizontal or vertical plane can be given in a closed form in one plane either knowing (a) the beam size and divergence at a minimum value of the size or (b) from any two values of beam radius and divergence measured in that plane. We then extend this later approach to determine emittance for the more general case of beams with space charge. Finally, we discuss the results of applying this approach to simulated beams with a prescribed value of emittance. 


\section{EMITTANCE MEASUREMENT FOR BEAMS WITH NEGLIGIBLE SPACE CHARGE}

Before we present the new technique, we briefly review an earlier method [14], in which the size and divergence data at the minimum of the quad-scan are used to directly calculate the emittance.

\section{A. Emittance from quad-scans}

In Ref. [14] it is shown that for an emittance-dominated beam, the cross-correlation term can be determined at the minimum of a quadrupole scan of the beam radius or divergence as a function of focusing strength. The method in that reference starts with writing the beam moments at the screen in terms of the moments at the lens. Then, they showed that at the focal length where rms beam size is minimum, the cross-correlation term can be simply derived from the rms beam size at the screen and the drift length. Here, we follow a different approach to derive a relation for the cross-correlation term which can be extended to beams with space charge.

Figure 1 shows a typical quadrupole scan setup. The focal length of the quadrupole (or solenoid) is scanned to achieve a minimum spot size on the screen located downstream at a distance $L$ from the quadrupole. Assuming no space charge, the rms transverse beam envelope in the drift region can be expressed as

$R(s)=\left[R_{0}{ }^{2}+2 \cdot R_{0} \cdot R_{0}^{\prime} \cdot s+\left(\frac{\epsilon^{2}}{{R_{0}{ }^{2}}^{2}}+R_{0}^{\prime 2}\right) \cdot s^{2}\right]^{1 / 2}$,

where $R_{0}$ and $R_{0}^{\prime}$ denote the initial $2 \times$ rms radius and slope of the envelope at the lens, $\epsilon$ is the effective emittance, and $s$ is the distance from the lens on the axis along the beam direction. Here, the radius $R$ stands for either of the two transverse radii, and does not assume the beam is

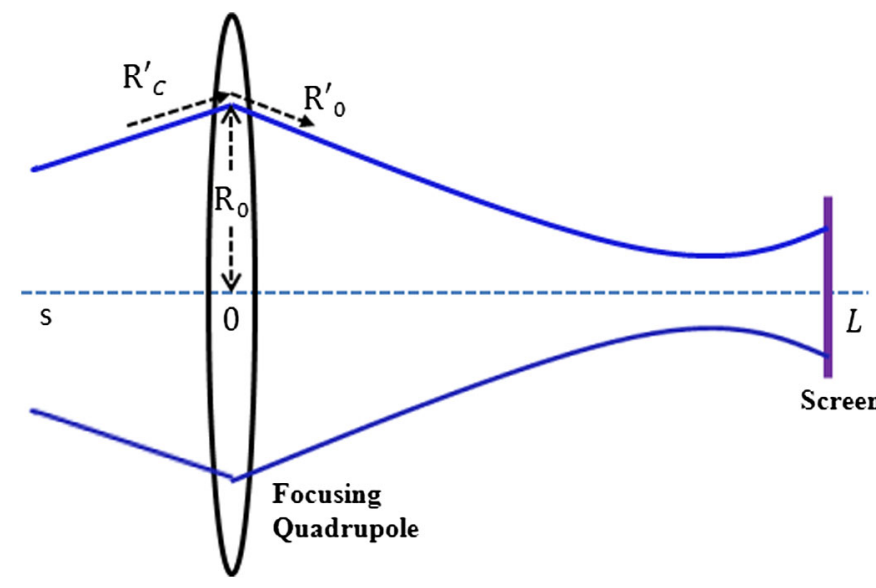

FIG. 1. Diagram of a quadrupole scan setup to measure emittance. Beam enters the quadrupole with envelope $R_{0}$ and slope $R_{C}^{\prime}$ and leaves it with slope $R_{0}^{\prime}$. axisymmetric. Then for beam radius at the location of screen we have

$$
R(L)=\left[R_{0}^{2}+2 \cdot R_{0} \cdot R_{0}^{\prime} \cdot L+\left(\frac{\epsilon^{2}}{{R_{0}}^{2}}+R_{0}^{\prime 2}\right) \cdot L^{2}\right]^{1 / 2} .
$$

The focal length required to have minimum beam radius on screen, $f_{m}$, can be found by setting the derivative of $R(L)$ with respect to $f$ equal to 0 . As according to Eq. (A3) in the Appendix, variations in $f$ simply translate to variations in beam slope right after the lens, $R_{0}^{\prime}$, we do the derivation with respect to $R_{0}^{\prime}$. The solution is

$$
\left.R_{0}^{\prime}\right|_{f=f_{m}}=-\frac{R_{0}}{L} .
$$

By plugging Eq. (3) into Eq. (1) the minimum beam radius at the screen is obtained as

$$
R_{\min }(L)=\frac{\epsilon \cdot L}{R_{0}} .
$$

To find the cross-correlation term we also need to calculate $R_{m}{ }^{\prime}(L)$, the slope of the envelope at the screen when the radius is minimum. Note that this is nonzero since the minimum radius does not correspond to a waist, being defined when $d R / d z=0$, whereas the minimum radius in a quad-scan occurs when $d R / d f=0$. Taking the derivative of $R(s)$ given in Eq. (1) with respect to $s$ and evaluating it at $s=L$, we obtain

$$
R^{\prime}(L)=\frac{R_{0} \cdot R_{0}^{\prime}+\left(\frac{\epsilon^{2}}{R_{0}{ }^{2}}+R_{0}^{\prime}{ }^{2}\right) \cdot L}{R(L)} .
$$

By substituting $R_{0}^{\prime}$ and $R(L)$ from Eqs. (3) and (4) we get

$$
R_{m}^{\prime}(L)=\frac{R_{\min }(L)}{L} .
$$

$R^{\prime}(L)$, alternatively, can be expressed as

$$
R^{\prime}(L)=2 \frac{d \sqrt{\left\langle r_{L}^{2}\right\rangle}}{d s}=2 \frac{\left\langle r_{L} \cdot r_{L}^{\prime}\right\rangle}{\sqrt{\left\langle r_{L}^{2}\right\rangle}}=4 \frac{\left\langle r_{L} \cdot r_{L}^{\prime}\right\rangle}{R(L)},
$$

where $r_{L}$ and $r_{L}^{\prime}$ denote the trace space $x$ or $y$ position and velocity of the particles at the screen. In case $x$, applying Eq. (7) to beam screen values at minimum radius and combining it with Eq. (6) leads to

$$
\left\langle x_{L} \cdot x_{L}^{\prime}\right\rangle=\frac{\left\langle x_{L}^{2}\right\rangle}{L} .
$$

Thus, the cross-correlation term can be determined from a single value of the beam size, i.e., its minimum measured value, and the $x$ rms emittance can be written in terms of the measured rms beam radius, $\sqrt{\left\langle x_{L}^{2}\right\rangle}$, and $\mathrm{rms}$ beam divergence, $\sqrt{\left\langle\dot{x}_{L}^{2}\right\rangle}$, as 


$$
\tilde{\boldsymbol{\epsilon}}_{x}^{2}=\left\langle x_{L}^{2}\right\rangle\left(\left\langle x_{L}^{\prime 2}\right\rangle-\frac{\left\langle x_{L}^{2}\right\rangle}{L^{2}}\right)
$$

Likewise, $\tilde{\epsilon}_{y}^{2}$ can be computed from vertical ( $y$ ) measurements. These expressions match those given in Ref. [14].

In summary, the envelope is measured over a range of quadrupole focusing strengths. Then, after fitting a quadratic to the data, $f_{m}$ and the rms radius at minimum are inferred from the curve. With the additional measurement of the rms divergence at $f_{m}$, the emittance is calculated from Eq. (9). Since we are dealing with an emittancedominated beam here, the advantage of this approach, in comparison to conventional quadrupole scan fitting, is that only one pair of simultaneously measured observables (size and divergence) are required to provide the emittance. However, as we will show the approach outlined above can be readily extended to provide a method to determine the emittance of a space-charge-dominated beam by using two pairs of values of the divergence and beam size measured along the quad-scan, i.e. without requiring that they include the minimum value of any of these parameters.

\section{B. Emittance from two samples of size and divergence}

In this section, we present a method for determining rms emittance from rms radius and divergence measured for two arbitrary settings of the quadrupole focusing strength. It is assumed that the following two sets of rms beam radii and divergences have been measured at two distinct focal lengths $f_{1}$ and $f_{2}$ :

$$
f_{1}\left\langle x_{1}^{2}\right\rangle\left\langle x_{1}^{\prime 2}\right\rangle, \quad f_{2}\left\langle x_{2}^{2}\right\rangle\left\langle x_{2}^{\prime 2}\right\rangle .
$$

From these measurements, we infer the cross-correlation terms, $X C_{i}(i=1,2)$. First, we note that $\tilde{\epsilon}^{2}$ can be written in terms of beam parameters at each of the focal settings as

$$
\tilde{\epsilon}^{2}=\left\langle x_{i}^{2}\right\rangle\left\langle x_{i}^{\prime 2}\right\rangle-X C_{i}^{2} \quad(i=1,2) .
$$

This leads to

$$
X C_{2}^{2}=X C_{1}^{2}+A
$$

where $A$ is defined as

$$
A \equiv\left\langle x_{2}^{2}\right\rangle\left\langle x_{2}^{\prime 2}\right\rangle-\left\langle x_{1}^{2}\right\rangle\left\langle x_{1}^{\prime 2}\right\rangle
$$

We find a second relation between the two crosscorrelation terms. To do so, we need to express $R_{0}$, the initial beam radius, in terms of beam parameters at the screen. It can be noticed that if the beam starts with radius $R(L)$ and slope $-R^{\prime}(L)$ at screen and propagates back toward the lens, then it obtains the radius $R_{0}$ and slope $R_{0}^{\prime}$ after traversing the drift. In other words, to derive $R_{0}$, we can switch $R_{0}$ and $R(L)$, and replace $R_{0}^{\prime}$ with $-R^{\prime}(L)$ in Eq. (2) to obtain

$R_{0}{ }^{2}=R(L)^{2}-2 \cdot R(L) \cdot R^{\prime}(L) \cdot L+\left(\frac{16 \tilde{\epsilon}^{2}}{R(L)^{2}}+R^{\prime}(L)^{2}\right) \cdot L^{2}$.
By substituting for $R^{\prime}(L)$ from Eq. (7) and simplifying the expression, one obtains $R_{0}$ in terms of rms values as

$$
R_{0}^{2}=4\left(\left\langle x_{i}^{2}\right\rangle-2 \cdot X C_{i} \cdot L+\left\langle x_{i}^{\prime 2}\right\rangle \cdot L^{2}\right) .
$$

Likewise, we can express $R_{0 i}^{\prime}(i=1,2)$, the envelope slope right after the lens, in terms of beam parameters at the lens. This time we switch $R_{0}$ and $R(L)$, and replace $R_{0}^{\prime}$ with $-R^{\prime}(L)$ in Eq. (5) to obtain

$$
R_{0 i}^{\prime}=-4 \frac{\left\langle x_{i}^{\prime 2}\right\rangle \cdot L-X C_{i}}{R_{0}}
$$

Using Eq. (A3) of the Appendix, we can express $f_{i}$, the focal length of the lens as

$$
\frac{1}{f_{i}}=\frac{R_{C}^{\prime}-R_{0 i}^{\prime}}{R_{0}}=\frac{R_{C}^{\prime} \cdot R_{0}+4\left\langle x_{i}^{\prime 2}\right\rangle \cdot L-4 X C_{i}}{R_{0}{ }^{2}} .
$$

Subtracting the two equations of (16) and substituting for $R_{0}^{2}$ in the denominator from Eq. (14) at $i=1$ (for measurement set 1), we obtain the following relation between two cross-correlation terms:

$$
X C_{2}=B \cdot X C_{1}+C
$$

with $B$ and $C$ defined as

$B \equiv 1-2 L\left(\frac{1}{f_{1}}-\frac{1}{f_{2}}\right)$,
$C \equiv\left(\left\langle x_{1}^{2}\right\rangle+\left\langle x_{1}^{\prime 2}\right\rangle \cdot L^{2}\right)\left(\frac{1}{f_{1}}-\frac{1}{f_{2}}\right)-\left(\left\langle x_{1}^{\prime 2}\right\rangle-\left\langle x_{2}^{\prime 2}\right\rangle\right) \cdot L$.

Combining Eqs. (11) and (17), we get following quadratic equation for $X C_{1}$ :

$\left(B^{2}-1\right) \cdot X C_{1}^{2}+2 \cdot B \cdot C \cdot X C_{1}+C^{2}-A=0$.

Therefore $X C_{1}$ can be obtained as

$X C_{1}=\frac{-B \cdot C \pm \sqrt{A \cdot B^{2}+C^{2}-A}}{B^{2}-1}, \quad B \neq \pm 1$.

By plugging $X C_{1}$ into Eq. (10), rms emittance can be calculated. It should be pointed out that canceling $R_{C}^{\prime}$ is the main reason for needing two pairs of measurements instead of just one. We could also form the second equation between cross correlations by using Eqs. (14), i.e. by matching the $R_{0}$ for two focal settings. For a thin lens, we should get the same answer whether we constrain the $R_{0}$ or the focal lengths $f_{1}$ and $f_{2}$ as we did above, however, for a real thick lens $R_{0}$ varies by changing the focusing strength of the lens. Therefore, a more accurate result is gained by constraining the focal lengths through Eq. (16).

As can be noticed, the method described here relies on two pairs of beam size and divergence samples to solve for the cross-correlation term and consequently the emittance, while the common Courant-Snyder parameter fitting technique requires at least three samples of beam size (or divergence) [2]. In comparison to our first approach, we do not require that the quadrupole scan curve go through a 
quadratic minimum - this can be a valuable advantage considering the fact that practically, the minimum may not be approachable. In addition we require divergence and size measurements for two distinct focal lengths to determine the emittance. Obviously, however, we can use multiple pairs to improve the statistical accuracy of the measurement.

We now extend the approach we have followed in this section to the universal case when space charge is not negligible.

\section{MEASURING EMITTANCE FOR BEAM WITH SPACE CHARGE}

For a uniform beam distribution where space charge is linear, the beam envelope evolution in a drift region is described by the following pair of coupled nonlinear ordinary differential equations (ODE):

$$
R_{j}^{\prime \prime}(s)-\frac{2 K}{R_{x}(s)+R_{y}(s)}-\frac{\epsilon_{j}^{2}}{R_{j}(s)^{3}}=0,
$$

where $j$ ranges over transverse coordinates $x$ and $y$, and dimensionless quantity

$$
K=\frac{q I_{b}}{2 \pi \epsilon_{0} m(c \beta \gamma)^{3}}
$$

is defined as the generalized perveance representing space-charge defocusing forces. In Eq. (22), $q$ is charge of the beam particles and $I_{b}$ is the beam peak current. Equation (21), also known as the KapchinskijVladimirskij (KV) envelope equation, is consistent with the KV phase space distribution which is an equilibrium solution of the Vlasov equation [15].

Because of the nonlinearity of the envelope equations in this case, a closed-form solution similar to Eq. (1) cannot be given. As before, we use a lens-drift-screen setup; however, due to the coupled nature of Eq. (21) we consider two separate cases based on beam cross section right before the lens and type of the lens: a round beam with a symmetric focusing lens, and an elliptical beam with either lens type or a round beam with an asymmetric focusing lens.

A. Round beam with symmetric focusing lens.-If the round beam with $R_{x}=R_{y}=R$ enters a symmetric focusing lens such as a solenoid then the beam maintains its roundness throughout the drift section and therefore we get two independent equations for both $R_{x}$ and $R_{y}$ :

$$
R_{j}^{\prime \prime}(s)-\frac{K}{R_{j}(s)}-\frac{\epsilon_{j}^{2}}{R_{j}(s)^{3}}=0 .
$$

Later in this section, we will present our scheme based on this case.

B. Elliptical beam with either lens type or round beam with asymmetric focusing lens.-For these asymmetric cases, Eq. (21) should be solved for both $R_{x}$ and $R_{y}$. A quadrupole is an example of an asymmetric focusing lens. A quadrupole or an elliptical beam manifests themselves as different initial conditions for differential Eq. (21) and therefore the beam hits the screen with different radius and divergence values for $x$ and $y$. Measuring emittance in such cases will be discussed later.

Returning to case A, we present a method for deriving emittance from two samples of beam radius and divergence. Since $R_{x}$ and $R_{y}$ are equal in this case, we drop the index $j$ and treat all parameters for $x$.

Multiplying Eq. (23) by $R^{\prime}$ and then integrating with respect to $s$ leads to the following first order ODE:

$R^{\prime}(s)= \pm\left[R_{0}{ }^{2}+\epsilon^{2} \cdot\left(\frac{1}{R_{0}{ }^{2}}-\frac{1}{R(S)^{2}}\right)+2 K \cdot \ln \left(\frac{R(S)}{R_{0}}\right)\right]^{1 / 2}$,

where $R_{0}$ and $R_{0}^{\prime}$, again, denote the radius and slope of the envelope at the lens. Similar to the zero space-charge case, we need to have the beam envelope relation in terms of at-screen radius and slope quantities. This can be done simply by switching $R_{0}$ and $R_{0}{ }^{\prime}$ with beam quantities at the screen, $R(L)$ and $-R(L)^{\prime}$, and applying a minus to the left-hand side of Eq. (24). Such minuses are necessary as we are treating the envelope evolution along $-s$. Thus, envelope ODE in terms of envelope parameters at screen can be expressed as

$$
\begin{aligned}
R^{\prime}(s)= & \mp\left[R(L)^{\prime 2}+\epsilon^{2} \cdot\left(\frac{1}{R(L)^{2}}-\frac{1}{R(S)^{2}}\right)\right. \\
& \left.+2 K \cdot \ln \left(\frac{R(S)}{R(L)}\right)\right]^{1 / 2} \cdot
\end{aligned}
$$

This form of envelope ODE can easily be solved by numerical integration in packages like MATLAB [16].

As there is no closed-form solution to this equation for large $K$, we cannot provide a closed-form answer for emittance. However, based on the analysis of previous section for the negligible space-charge case, we have devised a numerical procedure to determine the emittance.

We start with a guess for cross-correlation term and try to infer focal setting applied to the lens by solving Eq. (25). Beam radius and divergence measurements at the screen are translated to $R(L)$ and $R(L)^{\prime}$ and therefore inferred focal length is going to be a function of the guessed cross correlation. We use the error between calculated focal length with actual focal length to correct our guess for cross correlation and reiterate the procedure. Finally, after several steps, as the inferred focal length converge toward the actual one we come within a close vicinity of the actual cross correlation.

\section{A. Numerical procedure}

The detailed steps of the procedure are described here. As before we need two sets of beam radius and divergence measurements at two distinct focal lengths $f_{1}$ and $f_{2}$ : 


$$
f_{1}\left\langle x_{1}^{2}\right\rangle\left\langle x_{1}^{\prime 2}\right\rangle, \quad f_{2} \quad\left\langle x_{2}^{2}\right\rangle\left\langle x_{2}^{\prime 2}\right\rangle .
$$

Henceforth, we use subscript $n$, denoting the step number, alone or besides the measurement numbers 1 or 2 on all parameters that change over subsequent steps.

(1) $X C 1_{1}$, initial guess for the cross-correlation term at $f_{1}$, is chosen according to the discussion in the next section.

(2) Using Eq. (11), $X C 2_{n}$, the cross-correlation term at $f_{2}$, is calculated as

$$
X C 2_{n}= \pm \sqrt{X C 1_{n}^{2}+A} .
$$

(3) Next, $\epsilon_{n}$ (not to be confused with normalized emittance) and $R_{i}^{\prime}(L)$ for two measurement sets $(i=1,2)$ are calculated as

$$
\begin{gathered}
\epsilon_{n}=4\left[\left\langle x_{1}^{2}\right\rangle \cdot\left\langle x_{1}^{\prime 2}\right\rangle-X C 1_{n}{ }^{2}\right]^{1 / 2}, \quad R_{1}^{\prime}(L)_{n}=2 \frac{X C 1_{n}}{\sqrt{\left\langle x_{1}^{2}\right\rangle}} \\
R_{2}^{\prime}(L)_{n}=2 \frac{X C 2_{n}}{\sqrt{\left\langle x_{2}^{2}\right\rangle}}
\end{gathered}
$$

Note that all three relations are in terms of $X C 1_{n}$.

(4) ODE Eq. (25) can now be solved for finding at-lens envelope radius and slope conditions of measurements 1 and 2:

$$
R(0)_{1 n}, \quad R^{\prime}(0)_{1 n}, \quad R(0)_{2 n}, \quad R^{\prime}(0)_{2 n} .
$$

(5) According to Eq. (A3), for $f_{1 n}$ and $f_{2 n}$ estimates of focal lengths we have

$$
f_{1 n}=\frac{R(0)_{1 n}}{R_{C}^{\prime}-R^{\prime}(0)_{1 n}}, \quad f_{2 n}=\frac{R(0)_{2 n}}{R_{C}^{\prime}-R^{\prime}(0)_{2 n}} .
$$

Canceling the unknown $R_{C}^{\prime}$ between these two equations and replacing $f_{2 n}$ with its final value $f_{2}$ leads to

$$
f_{1 n}=\frac{f_{2} \cdot R(0)_{1 n}}{R(0)_{2 n}+f_{2} \cdot\left[R^{\prime}(0)_{2 n}-R^{\prime}(0)_{1 n}\right]} .
$$

This equation is used to update $f_{1 n}$ at each step $n$. As before, the necessity for two pair of measurements arises from $R_{C}^{\prime}$.

(6) It can be easily checked that $f_{1 n}$ is a function of $X C 1_{n}$. i.e. $f_{1 n}=g\left(X C 1_{n}\right) . X C 1_{n}$ should be modified so that reiteration of the procedure from entry 2 makes $f_{1 n}$ closer to the target value $f_{1}$. In other words, $X C 1_{n}$ is zero of this equation:

$$
g\left(X C 1_{n}\right)-f_{1}=0 .
$$

Since the derivative of the function $g$ is not known, a modified form of Newton's method [17] was used to find $X C 1_{n}$ as zero of this equation. In the first step $(n=1)$, $X C 1_{2}$ is valued in the vicinity of $X C_{1}$ :

$$
X C 1_{2}=0.95 X C 1_{1} \text {. }
$$

We may need to pick $X C 1_{2}$ closer to $X C 1_{1}$ if $X C 1_{2}$ makes the right-hand side of Eq. (26) imaginary. After the first step $(n \geq 2), X C 1_{n}$ is updated according to

$X C 1_{n+1}=X C 1_{n}-\frac{X C 1_{n}-X C 1_{n-1}}{g\left(X C 1_{n}\right)-g\left(X C 1_{n-1}\right)} \cdot\left[g\left(X C 1_{n}\right)-f_{1}\right]$.

Interval halving is another numerical root finder method that can be used to update $X C 1_{n}$. After updating $X C 1_{n}$ either way, the procedure is repeated from entry 2 until $f_{1 n}$ converges with desired precision toward $f_{1}$. The process can also be stopped when the variation on emittance calculated at two consecutive steps is less than some $\delta \%$ of the calculated emittance, where delta is usually chosen between 1 and 10. Finally, beam effective emittance is the last $\epsilon_{n}$ calculated in entry 3 .

\section{B. Choosing an initial value for $X C 1$}

Generally, choosing an appropriate initial value is important for convergence problems. The initial value for $X C 1$ should satisfy two constraints: $\epsilon_{1}{ }^{2}>0$ and $X C 2_{1}{ }^{2}>0$, i.e. both quantities are real. The first condition sets a hard minimum on the absolute value of $X C 1$, while the second one specifies a soft upper bound if $A<0$. There will be no upper limit when $A \geq 0$. According to relations (26) and (27) for $X C 2_{n}$ and $\epsilon_{n}$, we have

$$
\left|X C 1_{1}\right|<\sqrt{\left\langle x_{1}^{2}\right\rangle\left\langle x_{1}^{\prime 2}\right\rangle}, \quad\left|X C 1_{1}\right| \geqslant \sqrt{-A} \quad \text { if } A<0 .
$$

We define interval $R_{X C}$ as the distance between the two bounds:

$$
R_{X C} \equiv \sqrt{\left\langle x_{1}^{2}\right\rangle\left\langle x_{1}^{\prime 2}\right\rangle}-\sqrt{-A} \quad \text { if } A<0
$$

and

$$
R_{X C} \equiv \sqrt{\left\langle x_{1}^{2}\right\rangle\left\langle x_{1}^{\prime 2}\right\rangle} \quad \text { if } A \geq 0 .
$$

$X C 1_{1}$ is chosen to be at the middle of this interval:

$$
X C 1_{1}=S_{1} \cdot \begin{cases}\sqrt{-A}+\frac{R_{X C}}{2} & \text { if } A<0 \\ \frac{R_{X C}}{2} & \text { if } A \geq 0,\end{cases}
$$

where $S_{1}$ getting values from $\{-1,+1\}$ determines the sign of $X C 1_{1}$. Usually, the sign of $X C 1_{1}$ is known beforehand; however, one may try the other case if the first choice of sign leads to a divergent solution.

In the interval halving method we need to determine the initial interval $I_{1}$ too. For the negative $A$, such an interval can be chosen as

$$
I_{1}=S_{1} \cdot\left[\sqrt{-A}+0.05 \cdot R_{X C} \quad \sqrt{\left\langle x_{1}^{2}\right\rangle\left\langle x_{1}^{\prime 2}\right\rangle-0.05 \cdot R_{X C}}\right]
$$


and for positive $A$

$$
I_{1}=S_{1} \cdot\left[0.05 \cdot R_{X C} \quad \sqrt{\left\langle x_{1}^{2}\right\rangle\left\langle x_{1}^{\prime 2}\right\rangle}-0.05 \cdot R_{X C}\right] .
$$

\section{Elliptical beam}

As discussed earlier, if the beam entering the lens is elliptical or the lens is a quadrupole then the evolution of the $x$ or $y$ envelopes can diverge. We extend the approach discussed for a round beam to cover such asymmetric cases.

First, similar to the round beam case, we multiply Eq. (21) by $R_{j}{ }^{\prime}$ and then integrate with respect to $s$ for $j$ ranging over $x$ and $y$. We receive the following coupled first order ODE's in terms of beam values at $s=L$ :

$$
\begin{aligned}
R_{x}^{\prime}(s)= & \mp\left[R_{x}^{\prime}(L)^{2}+\epsilon^{2} \cdot\left(\frac{1}{R_{x}(L)^{2}}-\frac{1}{R_{x}(S)^{2}}\right)\right. \\
& \left.+4 K \cdot \ln \left(\frac{R_{x}(S)+R_{y}(S)}{R_{x}(L)+R_{y}(S)}\right)\right]^{1 / 2}, \\
R_{y}^{\prime}(S)= & \mp\left[R_{y}^{\prime}(L)^{2}+\epsilon^{2} \cdot\left(\frac{1}{R_{y}(L)^{2}}-\frac{1}{R_{y}(S)^{2}}\right)\right. \\
& \left.+4 K \cdot \ln \left(\frac{R_{y}(S)+R_{x}(S)}{R_{y}(L)+R_{x}(S)}\right)\right]^{1 / 2},
\end{aligned}
$$

where we have assumed that $x$ and $y$ emittances are about the same. Obviously, to solve these equations we need beam quantities in the $y$ plane as well. Thus, two sets of beam radius and divergence measurements at two distinct focal lengths $f_{1}$ and $f_{2}$ are going to be

$$
f_{1}\left\langle x_{1}^{2}\right\rangle,\left\langle y_{1}^{2}\right\rangle\left\langle x_{1}^{\prime 2}\right\rangle,\left\langle y_{1}^{\prime 2}\right\rangle, \quad f_{2}\left\langle x_{2}^{2}\right\rangle,\left\langle y_{2}^{2}\right\rangle\left\langle\left\langle x_{2}^{\prime 2}\right\rangle,\left\langle y_{2}^{\prime 2}\right\rangle .\right.
$$

We can follow the same procedure as before, except that envelope slopes in $y$ should be measured at entry 3 after calculating the emittance. To this end, we first calculate the cross correlations in $y$ for two measurements 1 and 2 according to

$$
\begin{aligned}
& Y C 1_{n}=4\left[\epsilon_{n}{ }^{2}-\left\langle y_{1}^{2}\right\rangle \cdot\left\langle y_{1}^{\prime 2}\right\rangle\right]^{1 / 2}, \\
& Y C 2_{n}=4\left[\epsilon_{n}{ }^{2}-\left\langle y_{2}^{2}\right\rangle \cdot\left\langle y_{2}^{\prime 2}\right\rangle\right]^{1 / 2},
\end{aligned}
$$

and then slopes can be calculated as

$$
R^{\prime}{ }_{y 1}(L)_{n}=4 \frac{Y C 1_{n}}{\sqrt{\left\langle y_{1}^{2}\right\rangle}}, \quad R_{y 2}^{\prime}(L)_{n}=4 \frac{Y C 2_{n}}{\sqrt{\left\langle y_{2}^{2}\right\rangle}} .
$$

To numerically solve ODE in (38), both equations are integrated simultaneously in entry 4 . It can be easily checked that by converging $X C 1_{n}$ toward its actual value the other three cross-correlation terms converge toward their actual values as well.

\section{SIMULATION RESULTS}

In this section, we present results of tests of our proposed approach with simulated beams. We used the code WARP $[18,19]$ to simulate the lens-drift experiment. Based
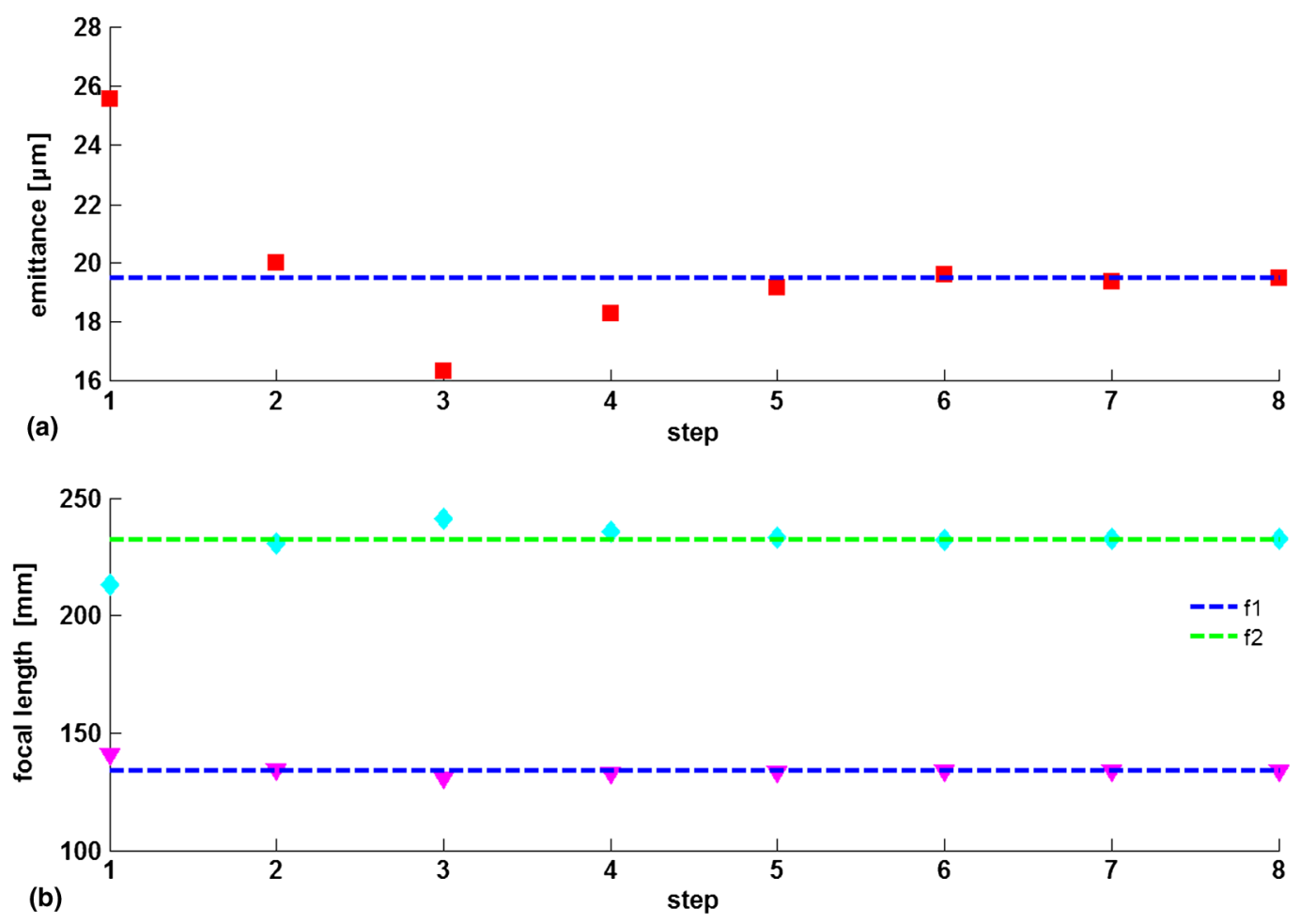

FIG. 2. Plots show convergence of emittance (a) and focal lengths $f_{1}$ and $f_{2}$ (b) for a low space-charge beam with $K=8.82 \times 10^{-5}$. The dashed line in (a) indicates the actual emittance while squares indicate the calculated emittance at each step. 

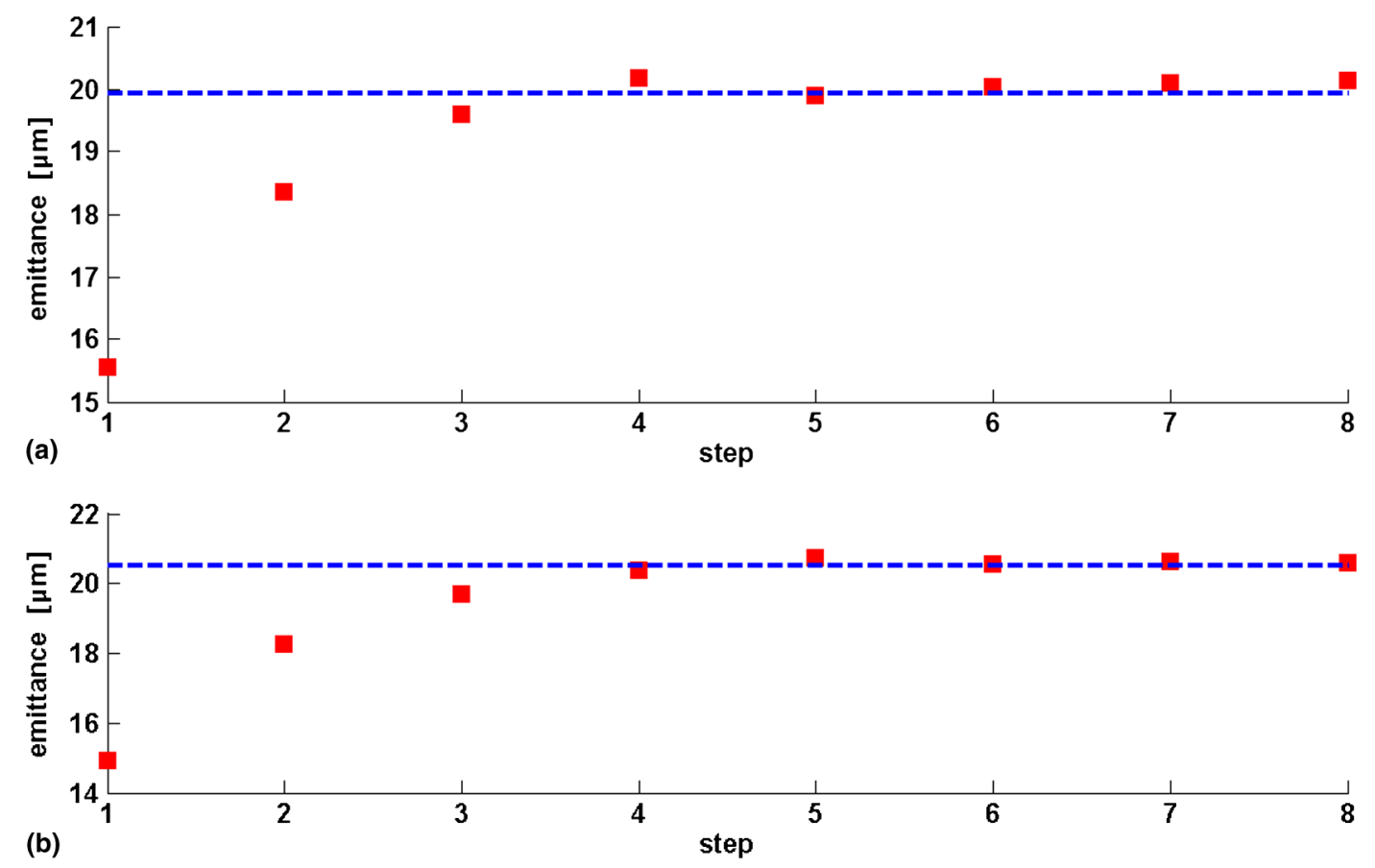

FIG. 3. Plot showing convergence of emittance for (a) a medium space-charge beam $\left(K=2.83 \times 10^{-4}\right)$, and (b) for a high space-charge beam $\left(K=7.06 \times 10^{-4}\right)$.

on presumed beam parameters $\epsilon$ and $K$ and an initial envelope radius at the lens, $R_{0}$, WARP generates a set of beam radius and divergence rms values $\left\langle x^{2}\right\rangle$ and $\left\langle x^{\prime 2}\right\rangle$ at the end of the drift section with length $L$ where beam divergence and size are supposedly measured, e.g., using optical transition radiation (OTR) interferometry [10]. The focal length of the lens, $f$, is calculated according to a hard edge model for the lens. The performance of the method for several grades of the space charge was tested. First, a low space-charge beam with the following parameters,

$$
\begin{aligned}
K & =8.82 \times 10^{-5}, \quad \epsilon=19.5 \mu \mathrm{m}, \\
R_{0} & =3 \mathrm{~mm}, \quad L=200 \mathrm{~mm},
\end{aligned}
$$

was simulated with a solenoid. Two pairs of beam radius and divergence samples taken at focal lengths, $f_{1}=134 \mathrm{~mm}$ and $f_{2}=232 \mathrm{~mm}$, were

$$
\begin{array}{lll}
f_{1} & \sqrt{\left\langle x_{1}^{2}\right\rangle}=3.5 \mathrm{~mm} & \sqrt{\left\langle x_{1}^{\prime 2}\right\rangle}=1.3 \mathrm{mrad}, \\
f_{2} & \sqrt{\left\langle x_{2}^{2}\right\rangle}=4.2 \mathrm{~mm} & \sqrt{\left\langle x_{2}^{\prime 2}\right\rangle}=1.2 \mathrm{mrad} .
\end{array}
$$

These samples were fed into the procedure. Figure 2 shows convergence curves for emittance and also the focal lengths. As can be seen, the emittance converges to within $1 \%$ of its simulated value after just five iterations. Figures 3(a) and 3(b) show emittance convergence curves for space-charge-dominated beams with $K=2.83 \times 10^{-4}$ and $K=7.06 \times 10^{-4}$, respectively. Still, the convergence is fast and errors in calculation of emittance are satisfactory.

To study the performance of the procedure for an elliptical beam, a quadrupole was used to generate the samples. Figure 4 shows the emittance convergence curve for such an asymmetric beam.

To see effectiveness of the proposed procedure, we compared the emittance measured with our technique to

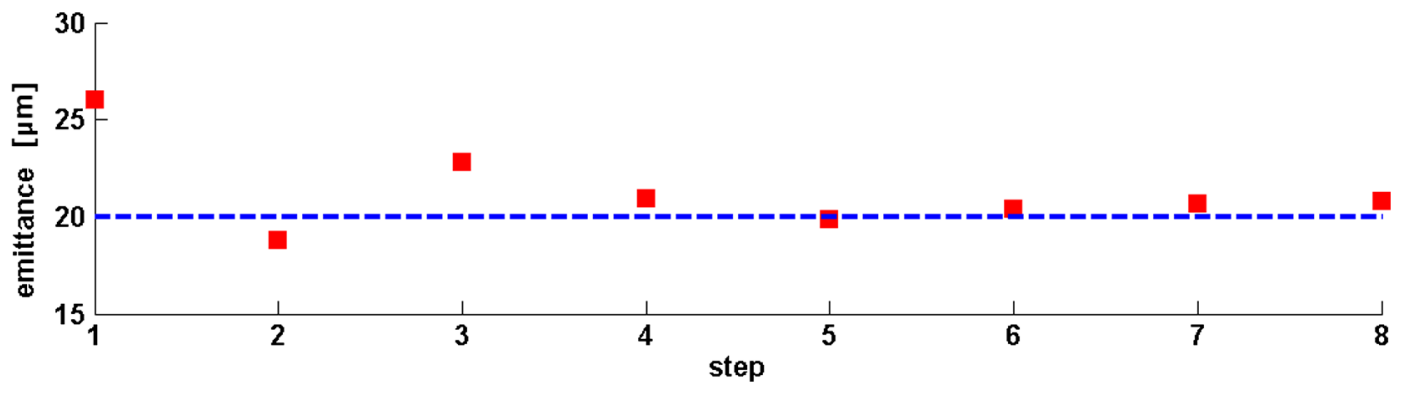

FIG. 4. Plot showing convergence of emittance for an elliptical beam with medium space charge $\left(K=2.83 \times 10^{-4}\right)$. 

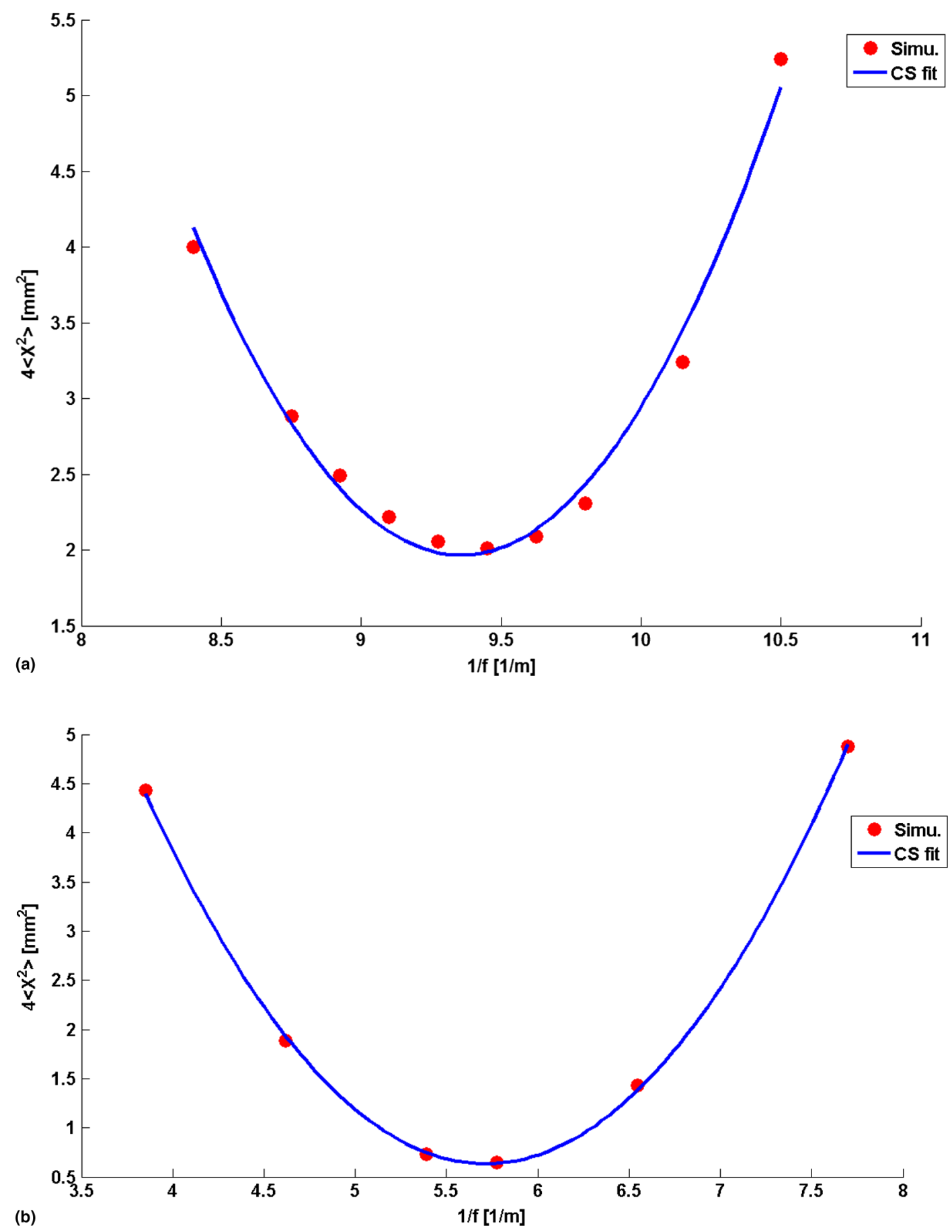

FIG. 5. Plot showing quadrupole radius scan for (a) beam with high space charge $\left(K=7.06 \times 10^{-4}\right)$ and (b) emittance-dominated beam $\left(K=7.35 \times 10^{-6}\right)$. The blue curve is a quadratic fit to the simulated data samples shown with red circles. The goodness-of-fit parameter is 0.95 for case (a) and 0.999 for case (b).

those obtained using other methods, i.e., the conventional Courant-Snyder (CS) parameter fitting and the minimum beam size method [14]. Figure 5(a) shows a scan of beam radius done for a high space-charge beam with $K=$ $7.06 \times 10^{-4}$. Note the poor quality of a quadratic fit to the data, represented by fairly low goodness-of-fit [20] value $R^{2}=0.95$, which is a sign that emittance measured by methods ignoring space charge are not reliable. In contrast, the quadratic fit $\left(R^{2}=0.99\right)$ shown in Fig. 5(b) for the emittance-dominated beam is excellent. In Fig. 6, we compare the emittance obtained with different methods as a function of the parameter $K$. As expected, the error in determining the emittance obtained using the conventional Courant-Snyder (CS) quadratic fitting technique and the 


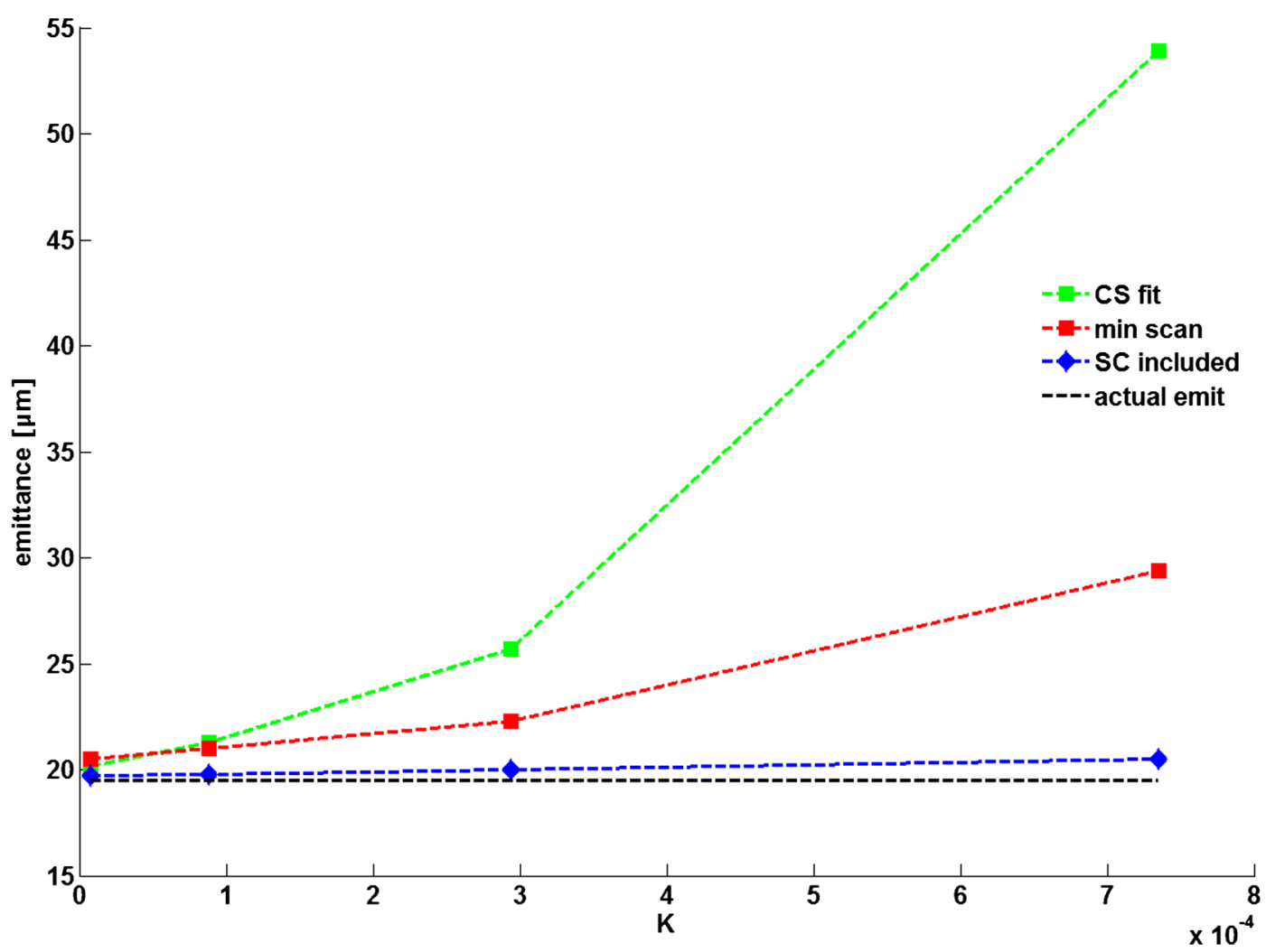

FIG. 6. Comparison of the emittance measured using different methods as a function of beam perveance $(K)$ : dashed green curve: CS parameter fitting method; red curve: cross-correlation determination at minimum of quad beam size scan; blue curve: two value method including space charge, showing small deviation from the actual emittance.

minimum beam size methods, which are both accurate for emittance-dominated beams, becomes increasing large as space charge increases. In contrast, our method gives acceptable values for the emittance for all values of the $K$ parameter shown.

In a real experiment there are some errors associated with the measurements. These errors will certainly lead to an error in the calculation of the cross correlation and consequently the emittance. The error is affected by the choice of the two focal lengths used to obtain the divergence and beam size. There are two criteria for selecting the focal strengths, $f_{1}$ and $f_{2}$. First, simple error analysis of the emittance relation shows that for a precise calculation, the cross-correlation term should not be larger than the emittance itself. This way, a $10 \%$ error in cross correlation translates roughly to the same $10 \%$ error in the emittance. If the cross correlation is about 3 times the emittance then the same $10 \%$ error in the cross correlation introduces a $30 \%$ error in the emittance. A good rule of thumb is to measure the emittance and see how it compares with the cross correlation. The result can be dismissed if the emittance is smaller. Second, the error is determined by how close $f_{2}$ is to $f_{1}$. Expectedly, choosing closely spaced focal lengths lead to large errors, and should be avoided. This can be easily checked from Eq. (23) for the negligible space-charge case. To limit the error gain due to the denominator of this equation one may choose $f_{2}$ according to

$$
\frac{1}{f_{1}}-\frac{1}{f_{2}}>\frac{1}{10 L}
$$

Figure 7 shows how an error in the measurement of beam divergence affects the emittance precision in the case of simulated high space-charge beam. The relative error in emittance is less than $17 \%$ for a $10 \%$ error in measurement of the divergence. As $f_{2}$ approaches $f_{1}$, the error increases and at $f_{2}=96 \mathrm{~mm}$ which corresponds to $\frac{1}{f_{1}}-\frac{1}{f_{2}} \sim \frac{1}{15 L}$ the error dramatically increases to more than $100 \%$.

Table I lists the errors in the calculation of emittance for various errors in measurement of the size and divergence. Overall, the approach shows robust response to errors in measurements.

Our theory makes use of the rms envelope equations to infer the emittance from two measurements of beam size and divergence which are obtained in the course of a magnetic quadrupole or solenoid scan. The underlying assumption is that the emittance is conserved between the lens and the screen. While such an assumption is fulfilled for many beam distributions such as $\mathrm{KV}$, Gaussian, and thermal (Maxwell-Boltzmann), the distribution function 


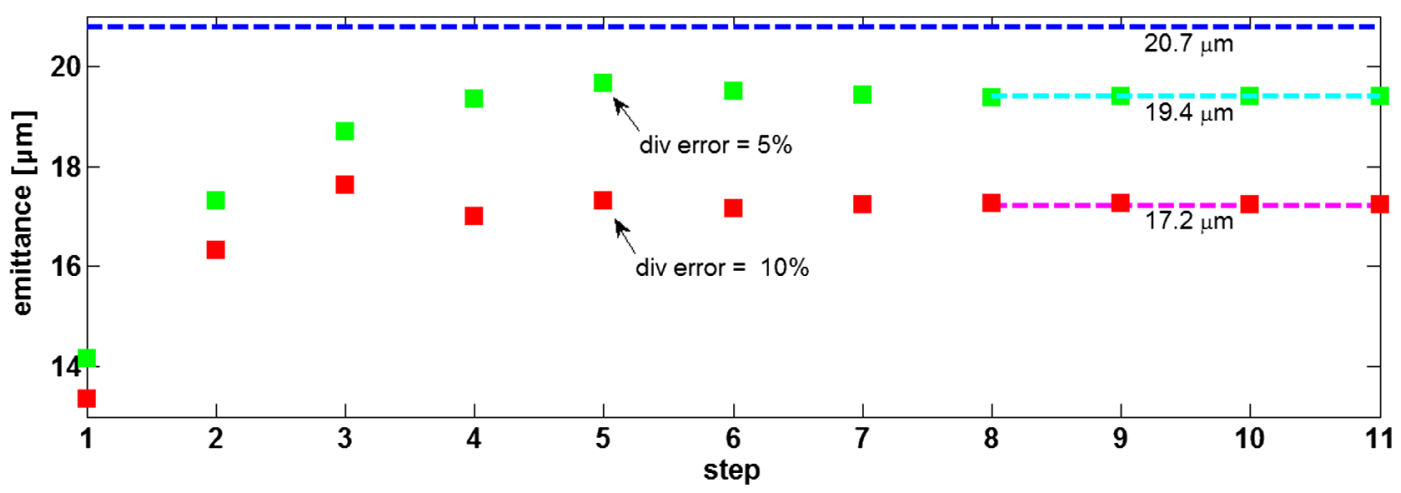

FIG. 7. Plot showing convergence of emittance for a round beam with high space charge $\left(K=7.02 \times 10^{-4}\right)$ when there are $5 \%$ and $10 \%$ errors in the divergence sampled at the focal length $f_{1}$.

for beams with strong space charge may be quite different. We expect then that the method we propose to calculate the emittance will not be accurate for beams with extremely high space charges, in particular when space-charge driven emittance growth is appreciable over the drift distance between the lens and the screen. To test the constant emittance assumption, we conducted self-consistent WARP simulations with different distributions. While the simulations test the distribution-dependent errors, for a particular accelerator there may be other sources of emittance growth that will introduce additional errors.

We have investigated the errors arising from the use of a linear space-charge model for the similar problem of tomographic phase space reconstruction [21]. The results of that investigation showed that the error is limited to $\sim 13 \%$ and fits the empirical formula:

$$
\text { error }(\%)=3+9.8 \chi^{2} \text {, }
$$

where $\chi$ is the intensity parameter. Note that this parameter is ill defined for a nonmatched beam over the drift distance of a quadrupole scan experiment. However, a calculation of $\chi$ at the lens, from

TABLE I. Errors in emittance with respect to beam measurement errors for the high space-charge beam $\left(K=7.02 \times 10^{-4}\right)$. Beam samples are taken at focal lengths $f_{1}=93 \mathrm{~mm}$ and $f_{2}=116 \mathrm{~mm}$.

\begin{tabular}{lc}
\hline \hline Measurement error & Relative error in emittance \\
\hline$\sqrt{\left\langle x_{1}^{2}\right\rangle}: 10 \%$ & $17 \%$ \\
$\sqrt{\left\langle x_{1}^{2}\right\rangle}: 5 \%$ & $6 \%$ \\
$\sqrt{\left\langle x_{1}^{\prime 2}\right\rangle}: 5 \%$ & $7 \%$ \\
$\sqrt{\left\langle x_{1}^{\prime 2}\right\rangle}: 10 \%$ & $17 \%$ \\
$\sqrt{\left\langle x_{2}^{2}\right\rangle}: 10 \%$ & $9 \%$ \\
$\sqrt{\left\langle x_{2}^{\prime 2}\right\rangle}: 10 \%$ & $10 \%$ \\
\hline \hline
\end{tabular}

$$
\chi=\frac{K}{K+\frac{\epsilon^{2}}{R(0)^{2}}}
$$

can be used to estimate the error.

Fortunately, there is a way to avoid such uncertainties in the measurements. Instead of using the $\mathrm{KV}$ envelope equations at entry 4 of our method, we can simulate the beam behavior with the initial condition given at entry 3 , and a distribution inferred from the beam imaging system. The procedure is continued from entry 5 by two pairs of $R(0)$ and $R^{\prime}(0)$ calculated from the simulations. By using a particle in cell (PIC) simulation code, like WARP, we can take into account any effect, such as a realistic model of the solenoid and image charge forces which is now considerable for a beam with extremely high space charge. Note that after convergence, the emittance obtained for the lens may be different than the screen emittance. Therefore, by replacing the envelope solver with the numerical simulator, we can always calculate the actual at-lens emittance regardless of how much it is different from the screen emittance. The results are reliable, as long as the simulator can give us accurate results for the beam distribution at the lens. Needless to say, it is less challenging for a simulator to give accurate results for the lens-drift-screen setup rather than the whole accelerator.

\section{CONCLUSION}

We have presented a novel approach to determine the rms emittance that includes space charge. In addition to the beam radius, the method also uses the beam divergence, both measurable, e.g. with OTR, in order to calculate the cross-correlation term and therefore the emittance. The method utilizes the similar lens-drift-screen setup used for a conventional quadrupole or solenoid scan. Two beam radius and divergence samples taken at two distinct focal strengths are then used in a procedure which computes the emittance in a progressive way. The approach involves taking the cross-correlation term as a control variable for matching beam envelopes at two focal settings 
to their actual envelopes under the constraint that the beam radii and divergences calculated, match their measured values at the position of the screen. We use a linear spacecharge model to solve for the beam envelope, and show that the errors in computation of the emittance are reasonably small when space-charge effects do not cause the emittance to vary significantly over the range of the scan.

In comparison, we showed that the method we present gives better measurement of the emittance for beams with significant space charge than conventional quad-scan fitting techniques.

\section{ACKNOWLEDGMENTS}

We are grateful for inspiring discussions with J.G. Power, C. F. Papadopoulos, and I. Haber. We also thank J. L. Vay and D. P. Grote for their outstanding support of the WARP code. The work is supported by the U.S. Department of Energy, Office of High Energy Physics Contract No. DEFG0207ER41489, the U.S. Department of Defense, Office of Naval Research Contract No. N000140711043 and the Joint Technology Office.

\section{APPENDIX: RELATION BETWEEN BEAM ENVELOPE PARAMETERS AT LENS WITH ITS FOCAL LENGTH}

According to Fig. 1, the beam envelope slopes at the entry and the exit of the lens represent particle trajectories tangent to the envelope right before and after the lens. Entry and exit beam coordinates are related by the transfer matrix of the converging thin lens with the focal length $f$ through

$$
\left[\begin{array}{l}
R_{0} \\
R_{0}^{\prime}
\end{array}\right]=\left[\begin{array}{cc}
1 & 0 \\
-\frac{1}{f} & 1
\end{array}\right] \times\left[\begin{array}{c}
R_{0} \\
R_{C}^{\prime}
\end{array}\right] .
$$

This leads to

$$
R_{0}^{\prime}=-\frac{R_{0}}{f}+R_{C}^{\prime}
$$

or alternatively

$$
\frac{1}{f}=\frac{R_{C}^{\prime}-R_{0}^{\prime}}{R_{0}}
$$

It should be noted that in a quad-scan experiment $R_{C}^{\prime}$ and $R_{0}$ remain unchanged throughout the scan process, while $R_{0}^{\prime}$ follows variations in $f$.
[1] B.E. Carlsten, J.C. Goldstein, P.G. O'Shea, and E. J. Pitcher, Nucl. Instrum. Methods Phys. Res., Sect. A 331, 791 (1993).

[2] C. Eckman et al., in Proceedings of the 3rd International Particle Accelerator Conference, New Orleans, Louisiana, USA, 2012 (IEEE, Piscataway, NJ, 2012).

[3] E. Chiadroni et al., in Proceedings of the DIPAC 2009, Basel, Switzerland (2009), pp. 402-404.

[4] R. Spesyvtsev et al., in Proceedings of the FEL 2008, Gyeongju, South Korea (2008).

[5] C. Thomas, N. Delerue, and R. Bartolini, JINST 6, P07004 (2011).

[6] S. G. Anderson, J. B. Rosenzweig, G.P. LeSage, and J. K. Crane, Phys. Rev. ST Accel. Beams 5, 014201 (2002).

[7] C.G. Limborg, S.M. Gierman, and J.G. Power, in Proceedings of the 20th Particle Accelerator Conference, Portland, OR, 2003 (IEEE, New York, 2003), pp. 2667-2669.

[8] P. A.P. Nghiem et al., in Proceedings of the 2nd International Particle Accelerator Conference, San Sebastián, Spain (EPS-AG, Spain, 2011), pp. 652-654.

[9] C. Lejeune and J. Aubert, in Advances in Electronics and Electron Physics, edited by A. Septier (Academic, New York, 1980), Supplement 13A, pp. 159-259.

[10] R. B. Fiorito and D. W. Rule, Optical Transition Radiation Beam Emittance Diagnostics, AIP Conf. Proc. No. 319 (AIP, New York, 1994), p. 21.

[11] A. Shkvarunets and R. Fiorito, Optical Synchrotron and Edge Radiation Diagnostics for Relativistic Electron Beams, AIP Conf. Proc. No. 732, edited by T. Shea and R. Shipley (AIP, New York, 2004), pp. 429-436.

[12] R. B. Fiorito, A.G. Shkvarunets, T. Watanabe, V. Yakimenko, and D. Snyder, Phys. Rev. ST Accel. Beams 9, 052802 (2006).

[13] M. Holloway, R. B. Fiorito, A. G. Shkvarunets, S. V. Benson, D. Douglas, P. Evtushenko, and K. Jordan, Phys. Rev. ST Accel. Beams 11, 082801 (2008).

[14] C. F. Papadopoulos, R. B. Fiorito et al., in Proceedings of the 23rd Particle Accelerator Conference, Vancouver, Canada, 2009 (IEEE, Piscataway, NJ, 2009).

[15] S. M. Lund and B. Bukh, Phys. Rev. ST Accel. Beams 7, 024801 (2004).

[16] The Mathworks Inc. [http://www.mathworks.com].

[17] G. J. Borse, Numerical Methods with MATLAB (PWS Publishing Company, Boston, 1997).

[18] A. Friedman, Nucl. Instrum. Methods Phys. Res., Sect. A 544, 160 (2005).

[19] http://hif.lbl.gov/theory/WARP_summary.html.

[20] Goodness-of-fit: http://www.mathworks.com/help/curvefit/ evaluating-goodness-of-fit.html.

[21] D. Stratakis, R. A. Kishek, I. Haber, S. Bernal, M. Reiser, and P. G. O'Shea, Phys. Rev. ST Accel. Beams 12, 064201 (2009). 